\title{
Thyroid Status, Iron, Folic Acid and Vitamin B12 Levels in Pregnancy
}

\author{
Manisha Baghel, Jyoti Batra, Thimmaraju Kv, Maliyanar Itagappa, \\ Sudhir Modala, Jai Singh Baghel. \\ ${ }^{1}$ Phd Scholar, Department Of Biochemistry Santosh Medical College, Ghaziabad, Delhi \\ ${ }^{2}$ Professor, Department Of Biochemistry Santosh Medical College, Ghaziabad, Delhi \\ ${ }^{3}$ Professor, Department Of Biochemistry Varunarjun Medical College, Shajahanpur, UP \\ ${ }^{4}$ Professor, Department Of Biochemistry Santosh Medical College, Ghaziabad, Delhi. \\ ${ }^{4}$ phd Scholar, Department Of Physiology Santosh Medical College, Ghaziabad, Delhi. \\ ${ }^{6}$ Medical Officer, Military Hospital, Jhansi.
}

\begin{abstract}
:
Background \& objectives: The role of iron deficiency with thyroid status in pregnancy was not studied in detail. Therefore, in this study, we assessed the levels of iron, folic acid, vitamin B 12, thyroid function and its association with iron levels in first trimester of pregnancy.

Materials and methods: Forty pregnant women who didn't start any supplementation were recruited from the obstetrics \& gynecology out patient department. Forty age matched controls were recruited from the residents and staff of the hospital. Thyroid profile, iron, folic acid and vitamin B12 levels were measured in both the groups. The association was seen between iron levels and thyroid stimulating hormone (TSH) levels.

Results: Levels of iron, folic acid, vitamin B12 are significantly low in first trimester pregnant women. Further, the increased TSH levels are negatively correlated with low iron levels.

Conclusion: Screening of iron, folic acid and vitamin B12 levels in first trimester itself will be beneficial to prevent the complications of pregnancy. Further, hypothyroidism also present and associated with iron deficiency. So, early diagnosis of these deficiencies will be useful to start giving supplements to avoid unwanted effects in pregnancy.
\end{abstract}

Keywords: Thyroid stimulating hormone, Vitamin B12, Folic acid, Plasma iron levels.

\section{Introduction}

Anatomical and physiological changes occur in the body of the mother during pregnancy to create a suitable environment for the growth of the fetus. A complex series of endocrinological and metabolic changes also takes place which facilitate the handling of nutrients by the body tissues of the mother as well as their transfer to the fetus [1]. Iron deficiency is the most commonly detected nutritional deficiency in pregnant women. During pregnancy, there is a significant increase in iron requirements due to increased red cell mass and fetoplacental growth [2]. Folic acid plays important role in fetal development [3] Folic acid plays a crucial role in the onecarbon metabolism for physiological nucleic acid synthesis and cell division, regulation of gene expression, amino acid metabolism and neurotransmitter synthesis [4]. During pregnancy, increased folate intake is required for rapid cell proliferation and tissue growth of the uterus and the placenta, growth of the fetus and expansion of the maternal blood volume [5]. Folate requirements are 5 to 10 fold higher in pregnant than in non-pregnant women [6], therefore pregnant women may be at risk for folate deficiency.

Vitamin B12 maintains normal folate metabolism which is essential for cell multiplication during pregnancy. Vitamin B12 deficiency is emerging as a growing public health problem and an increasing number of studies show that deficiency is commonly seen in pregnancy [7]. Pregnancy has a profound impact on the thyroid gland and thyroid function. The gland increases $10 \%$ in size during pregnancy in iodine-replete countries and by $20 \%-40 \%$ in areas of iodine deficiency. Production of thyroxine (T4) and triiodothyronine (T3) increases by $50 \%$, along with a $50 \%$ increase in the daily iodine requirement. These physiological changes may result in hypothyroidism [8].

However, the role of iron deficiency with thyroid status in pregnancy was not studied in detail. Therefore, in this study, we assessed the levels of iron, folic acid, vitamin b12, thyroid function and its association with iron levels in first trimester of pregnancy.

II. Materials And Methods

The study was carried out in the department of Biochemistry, Santosh Medical College \& Hospital, Ghaziabad (NCR). 
The procedures were explained to all subjects and written informed consent was obtained. The study protocol conformed to the ethical guidelines of the Declaration of Helsinki, as reflected in a prior approval by the institution's human research committee. Sample size was calculated with expected association between TSH and plasma iron will be 0.5 . In order to show that this is significantly different from 0 (no correlation) at alpha error 5\% (0.05) and power $80 \%$, with 2 sided test, we need to study 40 subjects in each group. Pregnant Group (PG=40), Control Group (CG=40).

The study was approved by Institute ethics committee. The study population was composed of pregnant attending the outpatient department of obstetrics and gynecology. The pregnant women coming for routine obstetrical evaluation in the first trimester of pregnancy, who had not initiated any kind of vitamin preparation and had no history of neural tube defect were included in the study. Participants with previous history of renal disease, alcohol consumption, evidence of malabsorption, smokers were excluded. Age matched controls are female staff and residents of the hospital. The exclusion criteria were same as in pregnant group.

Blood was collected after $8-10$ hours of fasting by venipuncture from all participants. Iron, vitamin B12 and folic acid measurements were performed using the chemiluminescence assay (Siemens ADVIA Centaur ${ }^{\circledR}$ immunoassay, Tarry town, NY, USA). Data expressed as mean \pm standard deviation (SD). To analyze the differences between groups, the independent student $t$ test was used. Pearson correlation was used to study the association of TSH with Iron status. Statistical analysis was performed using the R 3.2.3 for windows. P values less than 0.05 were considered statistically significant.

\section{Results}

One hundred and twenty four low-middle income pregnant women were recruited for the study. Eighty four were excluded from the study, since they had already started vitamin pills. The mean age and other baseline characteristics of study population and controls were depicted in Table 1. There was no significant differences in age, height and weight of pregnant and controls.

Table 1: Between comparison of anthropometric parameters.

\begin{tabular}{|l|l|l|l|}
\hline Sl.no & Parameter & Pregnant Group & Control Group \\
\hline 1 & Age (years) & $21.45 \pm 1.79$ & $22.05 \pm 2.43$ \\
\hline 2 & Height $(\mathrm{cms})$ & $155.45 \pm 5.56$ & $154.68 \pm 5.69$ \\
\hline 3 & Weight $(\mathrm{kg})$ & $67.50 \pm 6.90$ & $63.55 \pm 11.63$ \\
\hline 4 & HR (beats/min) & $80.35 \pm 1.80$ & $79.00 \pm 2.30 *$ \\
\hline 5 & SBP $(\mathrm{mmHg})$ & $116.85 \pm 5.69$ & $124.85 \pm 4.45 * * *$ \\
\hline 6 & DBP $(\mathrm{mmHg})$ & $75.20 \pm 3.19$ & $80.60 \pm 2.75 * * *$ \\
\hline
\end{tabular}

Data expressed as mean \pm SD. $*=p<0.005, * * *=p<0.000$.

Table 2. Shows the between group comparisons of iron, folic acid and vitamin B12 levels. There levels of these parameters are significantly less in pregnant when compared to healthy age matched controls. This was statistically significant $(\mathrm{p}<0.000)$.

Table 2: Between group comparison of iron, folic acid, vitamin B 12.

\begin{tabular}{|l|l|l|l|}
\hline Sl.no & Parameter & Pregnant Group & Control Group \\
\hline 1 & Iron (ug/dl) & $56.15+5.41$ & $86.15+18.08^{* * *}$ \\
\hline 2 & Folic acid (ng/ml) & $3.63+0.88$ & $5.78+0.33^{* * *}$ \\
\hline 3 & Vitamin B12 $(\mathrm{pg} / \mathrm{ml})$ & $198.43+24.41$ & $377.15+70.06 * * *$ \\
\hline
\end{tabular}

Data expressed as mean $\pm \mathrm{SD} . \quad * * *=\mathrm{p}<0.000$.

As depicted in Table 3, the TSH levels were significantly high in pregnants $(\mathrm{p}<0.000)$ when compared to controls. The fT3 and fT4 levels are significantly low in pregnant ( $\mathrm{p}<0.000)$. Further, the increased TSH levels were negatively correlated with reduced iron levels $(r=-0.309, p<0.052)$ (Table 4.)

Table 3: Between group comparisons of thyroid function tests.

\begin{tabular}{|l|l|l|l|}
\hline Sl.no & Parameter & Pregnant Group & Control Group \\
\hline 1 & TSH $(\mathrm{uIU} / \mathrm{ml})$ & $7.83+0.75$ & $3.61+0.82^{* * *}$ \\
\hline 2 & fT3 $(\mathrm{pg} / \mathrm{ml})$ & $0.87+0.38$ & $2.98+0.62 * * *$ \\
\hline 3 & fT4 $(\mathrm{ng} / \mathrm{dl})$ & $0.37+0.13$ & $1.36+0.28 * * *$ \\
\hline
\end{tabular}

Data expressed as mean \pm SD. $* * *=p<0.000$.

Table 4: Association of TSH and Iron.

\begin{tabular}{|l|l|l|l|}
\hline \multirow{2}{*}{ Sl. No } & Parameter & TSH $(\mathbf{u I U} / \mathrm{ml})$ \\
\cline { 2 - 4 } & & r value & p value \\
\hline $\mathbf{1}$ & Iron $(\mathbf{u g} / \mathbf{d l})$ & -0.309 & 0.052 \\
\hline
\end{tabular}




\section{Discussion}

Folate plays a crucial role in the one-carbon metabolism for physiological nucleic acid synthesis and cell division, regulation of gene expression, amino acid metabolism and neurotransmitter synthesis [4]. In this study, we found significantly lower levels of folic acid levels in first trimester of pregnancy when compared to age matched controls. This could be due to the fact that, during pregnancy, increased folate intake is required for rapid cell proliferation and tissue growth of the uterus and the placenta, growth of the fetus and expansion of the maternal blood volume. Folate requirements are 5- to 10-fold higher in pregnant than in non-pregnant women [6], therefore pregnant women may be at risk for folate deficiency.

Further, we found significantly lower levels of iron and vitamin B12 levels in first trimester of pregnancy when compared to controls. The first trimester pregnancies without any supplementation more likely reflect the preconceptional status. Our results emphasize that, although anemia is not a major problem, iron deficiency is a common problem in this geographical region. These pregnants will probably develop iron deficiency anemia with the progression of pregnancy. Screening in the preconceptional period or early pregnancy seems valuable for the detection and treatment of iron deficiency and anemia.

In this study, the increased TSH levels and decreased fT3 and fT4 levels indicates hypothyroidism. Earlier studies also reported that hypothyroidism is the most common pregnancy-related thyroid disorder, affecting $3-5 \%$ of all pregnant women. Subclinical hypothyroidism is more common than is overt hypothyroidism, and is usually defined as a serum TSH concentration greater than the pregnancy-specific reference range for each laboratory value, or by serum TSH concentrations greater than $2.5 \mathrm{mIU} / \mathrm{L}$ in the first trimester and greater than $3 \mathrm{mIU} / \mathrm{L}$ in the second and third trimesters. Some authors have defined subclinical hypothyroidism as a serum TSH between 5 and $10 \mathrm{mIU} / \mathrm{L}$, and overt hypothyroidism as a serum TSH greater than $10 \mathrm{mIU} / \mathrm{L}$, but this is not the commonly accepted definition. Once overt hypothyroidism is diagnosed, treatment with levothyroxine should be started to achieve serum TSH concentrations within the reference ranges for pregnancy as soon as possible [9].

However, the information about the association of iron deficiency with thyroid dysfunction was not well documented. The novelty of this study was, we studied the association of thyroid dysfunction with iron deficiency. We found significant negative correlation (Table. 4). This may be explained as, low iron, or more specifically, low ferritin, is one of the most overlooked causes of low thyroid function [10]. Ferritin is the stored form of iron that is used by the cells and a better measure of available iron levels than serum iron. Iron is a component of multiple enzymes involved with cellular metabolism, so low iron means poorly functioning enzymes, and less than optimal metabolism. Those with low iron also have low T4 and even lower T3 levels [11]. Iron deficiency also lowers thyroid peroxidase (TPO) activity. TPO is an iron-containing enzyme that initiates the first two steps in thyroid hormone synthesis.

\section{Conclusion}

From this study, we can conclude that, the iron, folic acid and vitamin B12 levels are low in first trimester itself. Further, hypothyroidism also present and associated with iron deficiency. So, early diagnosis of these deficiencies will be useful to start giving supplements to avoid unwanted effects in pregnancy.

Conflict of interest: None.

\section{References}

[1]. Mother and Child Nutrition in the Tropics and Subtropics - mcnts_chap4.pdf [Internet]. [cited 2015 Jul 11]. Available from: http://www.oxfordjournals.org/our_journals/tropej/online/mcnts_chap4.pdf

[2]. Allen LH. Pregnancy and iron deficiency: unresolved issues. Nutr Rev. 1997 ;55(4):91-101.

[3]. Scholl TO, Johnson WG. Folic acid: influence on the outcome of pregnancy. Am J Clin Nutr. 2000;71(5):1295s - 1303s

[4]. Djukic A. Folate-responsive neurologic diseases. Pediatr Neurol. 2007;37(6):387-97.

[5]. Rondo PH, Tomkins AM. Folate and intrauterine growth retardation. Ann Trop Paediatr. 2000;20(4):253-8.

[6]. Antony AC. In utero physiology: role of folic acid in nutrient delivery and fetal development. Am J Clin Nutr. 2007;85(2):598S $603 \mathrm{~S}$.

[7]. Sande HV, Jacquemyn Y, Karepouan N, Ajaji M. Vitamin B12 in pregnancy: Maternal and fetal/neonatal effects-A review. Open J Obstet Gynecol. 2013;03(07):599.

[8]. Stagnaro-Green A, Abalovich M, Alexander E, Azizi F, Mestman J, Negro R, et al. Guidelines of the American Thyroid Association for the Diagnosis and Management of Thyroid Disease During Pregnancy and Postpartum. Thyroid. 2011 ;21(10):1081-125.

[9]. Teng W, Shan Z, Patil-Sisodia K, Cooper DS. Hypothyroidism in pregnancy. Lancet Diabetes Endocrinol. 2013;1(3):228-37.

[10]. barb. Thyroid Hormone Requires Iron, Cortisol, Selenium, Iodine [Internet]. Tired Thyroid. [cited 2015 Jul 12]. Available from: http://www.tiredthyroid.com/cofactors.html

[11]. Hess SY, Zimmermann MB, Arnold M, Langhans W, Hurrell RF. Iron Deficiency Anemia Reduces Thyroid Peroxidase Activity in Rats. J Nutr. 2002;132(7):1951-5. 